\title{
Adsorptive Removal of Pyridine from Aqueous Solution Using Natural Shale
}

\author{
Yi Wu ${ }^{1,2}$, Kangle Ding1,2*, Mei Zou1,2, Zhenzhen Yu1, ${ }^{1,2}$, Chao Han ${ }^{1,2}$, Yan Liu ${ }^{3}$, Fujia Guan4 \\ ${ }^{1}$ Key Laboratory of Exploration Technologies for Oil and Gas Resources, Ministry of Education, Yangtze University, Wuhan \\ Campus, Wuhan, China \\ ${ }^{2}$ College of Chemistry and Environmental Engineering, Yangtze University, Jingzhou, China \\ ${ }^{3}$ College of Resources and Environment, Yangtze University, Wuhan Campus, Wuhan, China \\ ${ }^{4}$ College of Petroleum Engineering, Yangtze University, Wuhan Campus, Wuhan, China \\ Email: *dingkl@yangtzeu.edu.cn
}

How to cite this paper: Wu, Y., Ding, K.L., Zou, M., Yu, Z.Z., Han, C., Liu, Y. and Guan, F.J. (2021) Adsorptive Removal of Pyridine from Aqueous Solution Using Natural Shale. Open Journal of Yangtze Gas and Oil, 6, 36-49.

https://doi.org/10.4236/ojogas.2021.62004

Received: March 5, 2021

Accepted: April 19, 2021

Published: April 22, 2021

Copyright ( 2021 by author(s) and Scientific Research Publishing Inc. This work is licensed under the Creative Commons Attribution International License (CC BY 4.0).

http://creativecommons.org/licenses/by/4.0/ (c) (i) Open Access

\begin{abstract}
The discharge of pyridine bearing wastewater into water bodies without a prior satisfactory treatment would pose significant public health risk as well as serious threat to the aquatic ecosystems. In this study, a natural shale from Yichang, China is investigated to determine its potential as a low-cost adsorbent for trace pyridine removal from wastewaters. The prepared shale samples without surface modification are characterized by Fourier transform infrared spectroscopy (FT-IR), X-ray diffraction (XRD) and scanning electron microscope (SEM). Kinetics and isotherms of pyridine from aqueous solutions onto shale are investigated on the basis of the experimental data. It is found that the shale samples with well-developed porosity are mainly composed of illite, quartz, calcite, chlorite and sericite. Several kinetic models (viz. pseudo-first-order, pseudo-second-order, two-constant rate, intra-particle diffusion and Elovich) as well as isotherm models (Langmuir, Freundlich and Temkin) are applied to test the experimental data for pyridine removal. The kinetics of the adsorption of pyridine by shale follows a pseudo-second-order rate law with the adsorption data being best described by the Freundlich isotherm model. The preliminary study shows that natural shale obtained from sedimentary basins may be used as a potential low-cost adsorbent for the removal of trace pyridine from effluents.
\end{abstract}

\section{Keywords}

Natural Shale, Sedimentary Basins, Pyridine, Adsorption, Kinetics, Isotherms

\section{Introduction}

Pyridine is a common N-heterocyclic aromatic compound in effluents dis- 
charged from chemical factories, coking plants, pharmaceutical factories and the related industries [1]. As one of the priority pollutants listed by the United States Environmental Protection Agency [2], pyridine exhibits significant toxicity toward organisms and potential mutagenicity, teratogenicity as well as carcinogenicity to human beings [1] [3]. Especially, in comparison with other aromatic hydrocarbons pyridine is more difficult to be degraded by indigenous microorganisms, leading to its long-term stable existence in the environment. The discharge of pyridine bearing wastewater into water bodies without a prior satisfactory treatment would pose significant public health risk as well as serious threat to the aquatic ecosystems. In wastewater, the typical concentration of pyridine and its associated compounds may vary in the range of $20-300 \mathrm{mg} / \mathrm{L}$ [2] [4] [5]. A variety of physic-chemical treatment techniques have been developed for the removal of pyridine from wastewater, such as photocatalysis, ion exchange, extraction, foam fractionation, gamma irradiation, microwave radiation, electrochemistry, oxidation, biological treatment and adsorption. Among all the mentioned techniques, adsorption is one of the simplest, effective and economical method for the removal of low concentrations of organic pollutants from large volumes of potable water, process effluents, wastewater, and aqueous solutions [6]. Over the last decade, activated carbon (AC) has been proved to be effective for the removal of pyridine and its derivatives from industrial effluents [7] [8]. However, the disadvantage associated with AC lies in, the need for a costly regeneration system, the generation of carbons fines due to the brittle nature of carbons as well as its high initial manufacture cost [9] [10]. These make AC less economically viable as an adsorbent and led many investigators to search low-cost, naturally occurring adsorbents to remove trace pyridines from wastewater.

Yichang is located in central China, the southwest of Hubei Province, the upper and middle reaches of the Yangtze River. The shale in Yichang is a fine-grained sedimentary rock, generally characterized by large specific surface area and high porosity. Most previous studies are conducted with a focus on methane adsorption behavior on shale matrix [11]. To the best of our knowledge, there is no data available in literature about the removal of pyridines by shale. The objectives of this study are to:

1) Examine the feasibility of raw shale for pyridine removal from wastewater without any pretreatment procedures;

2) Study the adsorption characteristics, i.e. kinetics and isotherms of pyridine from aqueous solutions onto shale.

\section{Materials and Methods}

\subsection{Materials and Preparation of Adsorbents}

Pyridine, hydrochloric acid, sodium hydroxide and other chemicals are analytical reagents and purchased from Aladdin Industrial Corporation (Shanghai, China). Natural shale in this study is obtained from Yiye-1 shale gas well in Yichang, Hubei province of China at the burial depth of $1835.0 \mathrm{~m}$. The shale sam- 
ple is crushed with a mechanical rock crusher, ground in a ceramic mill, and then sieved to the desired particle size fractions. The sample of the particle size $177 \mu \mathrm{m}$ is washed using magnetic stirrer with the distilled water to remove any dissolved salts. After filtration, the washed sample is dried at $110^{\circ} \mathrm{C}$ overnight in an electric oven (Tianjin Taisite Instrument Co., Ltd., 101-1AB). The dry shale sample is kept in a desiccator over silica gel desiccant until use in characterization and adsorption experiments.

\subsection{Instruments}

The X-ray diffraction (XRD) technique is used to obtain the semi-qualitative mineralogical compositions of the shale samples. The samples are mechanically crushed and ground to size of $<200$ mesh. XRD data are collected at room temperature with a PANalytical X'Pert PRO X-ray diffractometer (PANalytical Co., Almelo, Netherlands). About $30 \mathrm{mg}$ of powdered solid samples are kept in a quartz block and pressed onto the quartz block using a glass slide to obtain a uniform distribution. The $2 \theta$ Bragg angles are scanned over a range of $5-85$ at a scanning speed of $6.25 / \mathrm{min}$, using graphite monochromated $\mathrm{Cu}-\mathrm{K}_{\alpha}$ radiation source and a nickel filter. The tube current and the tube voltage is $30 \mathrm{~mA}$ and 40 $\mathrm{kV}$, respectively. The experiment parameters are as followed: $\mathrm{DS}=1 / 4, \mathrm{SS}=1 / 8$, $\mathrm{RS}=5.0 \mathrm{~mm}$. The computer automates the data collection and data reduction steps of the analysis. To obtain a semiquantitative measurement of the mineral components of a given sample, the maximum intensity of each identified mineral is measured and compared to the standard intensity obtained from a pure mineral sample.

The FT-IR spectra are obtained in a diffuse reflectance infrared Fourier transform spectroscopy (DRIFTS) mode using the micro sampling cup of a Spectra-Tech diffuse reflectance accessory against a $\mathrm{KBr}$ background on a Nicolet 750 FT-IR spectrometer (Thermo Nicolet Corporation, Waltham, MA, DTGS detector; Nichrome source; $\mathrm{KBr}$ beam splitter). A small amount of the shale samples is finely ground, mixed with dried potassium bromide (Merck, Sharp and Dhome Ltd., Shanghai, China, spectroscopic grade) in the ratio of 1:100, and pressed into pellets for a spectrum test. The FT-IR spectra are collected and manipulated using the Thermo Electron software, OMNIC 7.0, supplied from the manufacturer of the spectrometer by averaging 32 scans at a spectral resolution of $4 \mathrm{~cm}^{-1}$ under a dried nitrogen flow $\left(10 \mathrm{~cm}^{3} / \mathrm{min}\right)$ condition. All spectra are smoothed using the "automatic smooth" function of the above software, which uses the Savitsky-Golay algorithm (95-point moving second-degree polynomial). After that, the baseline is corrected using the "automatic baseline correct" and the spectra scale is normalized with the "normalize scale" function. The spectroscopic region from $4,000 \mathrm{~cm}^{-1}$ to $400 \mathrm{~cm}^{-1}$ is used for the hierarchical cluster analysis.

Morphology of the shale samples is observed by a field emission-scanning electron microscope (FE-SEM, Model MIRA3 -XMU, TESCAN Czech Republic) operated at an acceleration voltage of $15 \mathrm{kV}$. The samples are observed under the 
conditions of $10.0 \mathrm{k}$ and $50.0 \mathrm{k}$ magnifications.

\subsection{Adsorption Procedure}

In batch adsorption experiments, accurately weighed shale samples $(0.1-1.5 \mathrm{~g})$ and $30 \mathrm{~mL}$ of the aqueous solution containing different initial concentrations of pyridine $(2.0-50 \mathrm{mg} / \mathrm{L})$ are put into a glass-stoppered flask. The flask is darkly brown-colored to prevent photoxidation. The $\mathrm{pH}$ of the solution is adjusted with $\mathrm{HCl}$ or $\mathrm{NaOH}$ solution by using a $\mathrm{pH}$ meter (Model PB-10, Shanghai Cany Precision Instrument Co., Ltd, China). The flask is subsequently capped with the glass stopper and shaken in a temperature-controlled water bath shaker (Model SHA-B, Tianjin Saidlis Experimental Analytical Instrument Factory, China) for $2 \mathrm{~h}$ at the temperature range of $25^{\circ} \mathrm{C}-45^{\circ} \mathrm{C}$. At preset contact times, the concentrations of pyridine in the solution are analyzed for residual concentration of pyridine at $\lambda_{\max }=256 \mathrm{~nm}$ [7] using a $722 \mathrm{~N} \mathrm{UV}$-vis spectrophotometer (Shanghai AuCy Scientific Instrument Co., Ltd., China). The amount adsorbed is calculated from the concentration of the pyridine solution before and after the adsorption experiments using a Beer's law plot to interpolate concentrations. Pyridine uptake at equilibrium, $q_{\mathrm{e}}(\mathrm{mg} / \mathrm{g})$, is calculated by the equation, $q_{\mathrm{e}}=\left(C_{0}-C_{\mathrm{e}}\right) V / W$. The $C_{0}$ and $C_{\mathrm{e}}(\mathrm{mg} / \mathrm{L})$ are the concentrations of pyridine at initial and at equilibrium, respectively. $V$ is the volume of the solution (L) and $W$ is the mass of dry adsorbent used (g). The calibration curves between absorbance and the concentration of the pyridine solution are established. The calibration plot of absorbance versus concentration of pyridine shows a linear variation. The standard curve is given as $y=31.895 x+0.002, \mathrm{R}^{2}=0.9994$, where $y$ is the absorbance and $x$ is the pyridine concentration.

\section{Results and Discussion}

\subsection{Characterization of Adsorbents}

The FT-IR spectra of the shale sample are given in Figure 1. The broad and strong band in the range of $3140-3620 \mathrm{~cm}^{-1}$ is assigned to the $-\mathrm{OH}$ stretching vibration in hydroxyl groups of constitution water and interlayer water of clay minerals [12]. The peak at $1410 \mathrm{~cm}^{-1}$ is attributed to the stretching vibration of $\mathrm{C}-\mathrm{O}$ in the carbonate anion $\left(\mathrm{CO}_{3}^{2-}\right)$ of calcite [13]. The intense band in the range of $1000-1100 \mathrm{~cm}^{-1}$ is ascribed to the $\mathrm{Si}-\mathrm{O}$ vibration in quartz [14]. The peaks at $515-795 \mathrm{~cm}^{-1}$ indicate the vibration of $\mathrm{Si}-\mathrm{O}, \mathrm{Al}-\mathrm{O}-\mathrm{H}$ and $\mathrm{Si}-\mathrm{O}-\mathrm{Al}$ [14].

X-ray diffraction patterns of the shale sample are exhibited in Figure 2. Quantitative mineralogical analyses by XRD (Figure 2) reveal that the typical shale sample primarily consists of illite (45.94 wt\%), quartz (38.05 wt\%), calcite (11.28 wt\%), chlorite (3.04 wt\%) and sericite (1.68 wt\%).

The SEM micrograph in Figure 3 is employed to evaluate the morphological structure of the shale. Figure 3 shows that the prepared the shale sample is characterized by various visible fractures and pore structures with different diame- 
ters, which may be derived from naturally and/or artificially occurring disintegration of the shale matrix. The well-developed porosity of the shale may favor pyridine removal during the actual effluent treatment process.

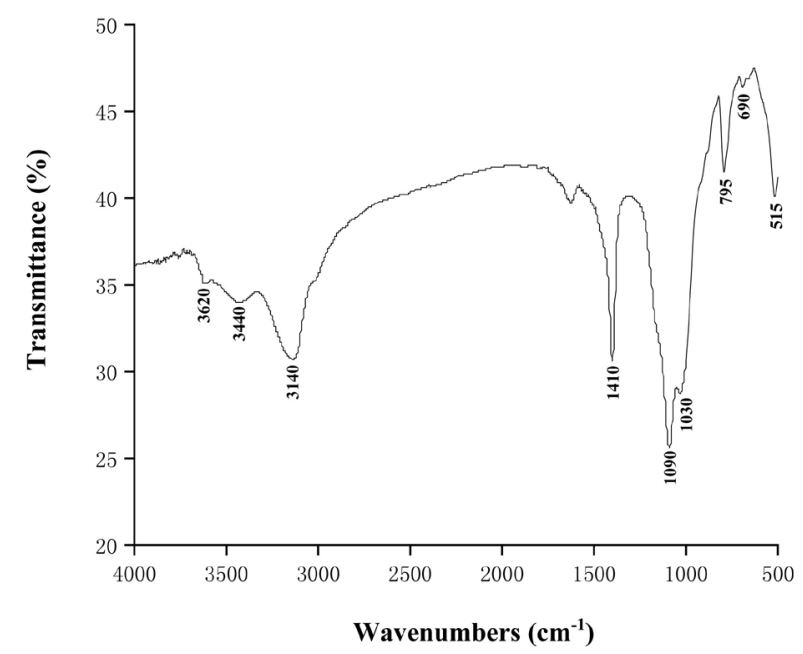

Figure 1. FT-IR spectra of the shale sample.

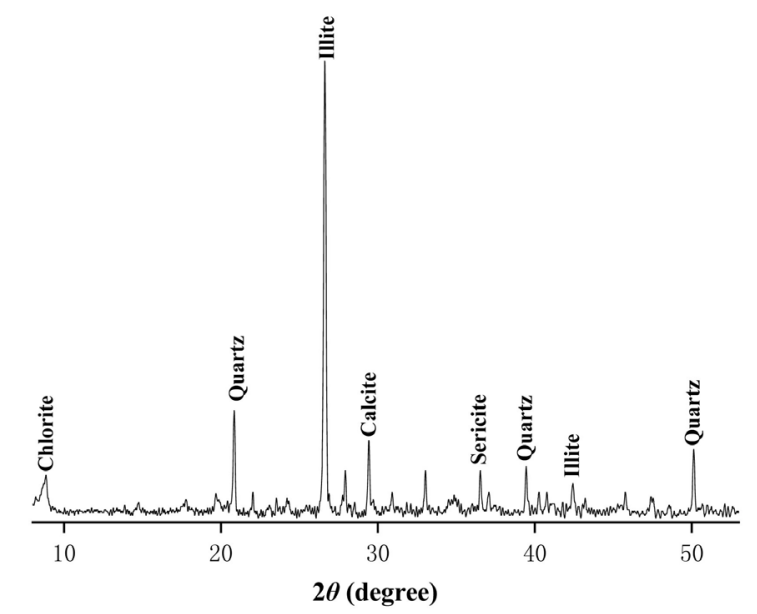

Figure 2. X-ray diffraction patterns of the shale sample.
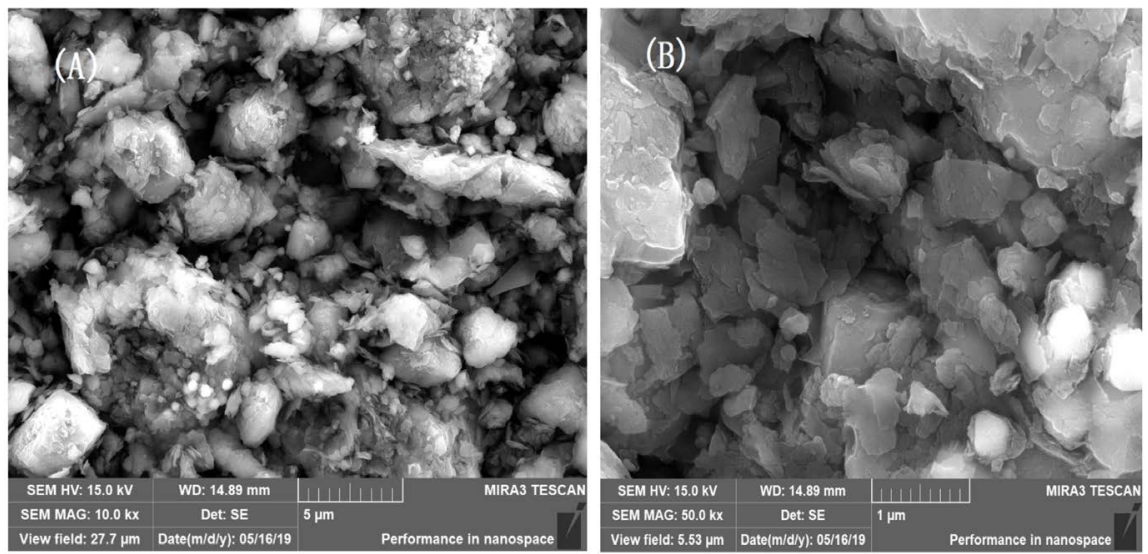

Figure 3. The SEM images of the shale sample at different magnifications. 


\subsection{Adsorption Kinetics}

The study of adsorption kinetics can provide valuable insights into the uptake rate of sorbents, the adsorption mechanisms as well as the proper design and model for the adsorption process. The experimental kinetic data of adsorption of pyridines onto the shale are analyzed by pseudo-first order, pseudo-second order, Elovich equation, intra-particle diffusion and two-constant rate equation [14], respectively. The fitting results are listed in Table 1. The pseudo first order model is the earliest developed equation that explains reversible equilibrium between adsorbate and adsorbent, assuming the rate in which the sites becoming occupied is exactly equal to number of vacant sites [14]. The pseudo-first-order rate equation is generally described by the following equation:

Table 1. Kinetic parameters of adsorption of pyridine on shale.

\begin{tabular}{|c|c|c|c|}
\hline Kinetic model & Parameters & $298 \mathrm{~K}$ & $\mathrm{R}^{2}$ \\
\hline Pseudo-first-order & $\begin{array}{l}q_{\mathrm{e}}\left(\mathrm{mg} \cdot \mathrm{g}^{-1}\right) \\
k_{1}\left(\min ^{-1}\right)\end{array}$ & $\begin{array}{l}0.516 \\
0.0169\end{array}$ & 0.891 \\
\hline Pseudo-second-order & $\begin{array}{c}q_{\mathrm{e}}\left(\mathrm{mg} \cdot \mathrm{g}^{-1}\right) \\
k_{2}\left(\mathrm{~g} \cdot \mathrm{mg}^{-1} \cdot \mathrm{min}^{-1}\right)\end{array}$ & $\begin{array}{l}0.530 \\
0.186\end{array}$ & 0.999 \\
\hline Two-constant & $\begin{array}{c}a\left(\mathrm{mg} \cdot \mathrm{g}^{-1} \cdot \mathrm{min}^{-1}\right) \\
b\left[\left(\mathrm{mg} \cdot \mathrm{g}^{-1}\right)^{-1}\right]\end{array}$ & $\begin{array}{l}0.309 \\
0.0904\end{array}$ & 0.970 \\
\hline Intra-particle diffusion & $\begin{array}{c}I\left(\mathrm{mg} \cdot \mathrm{g}^{-1}\right) \\
K_{\mathrm{id}}\left(\mathrm{mg}^{-1} \cdot \mathrm{g}^{-1} \cdot \mathrm{min}^{-0.5}\right)\end{array}$ & $\begin{array}{l}0.3831 \\
0.00794\end{array}$ & 0.899 \\
\hline Elovich & $\begin{array}{c}\alpha\left(\mathrm{mg}^{-1} \mathrm{~g}^{-1} \cdot \mathrm{min}^{-1}\right) \\
\beta\left(\mathrm{g} \cdot \mathrm{mg}^{-1}\right)\end{array}$ & $\begin{array}{l}33.239 \\
23.981\end{array}$ & 0.974 \\
\hline
\end{tabular}

where $q_{\mathrm{e}}$ and $q_{\mathrm{t}}$ are the amounts of pyridine adsorbed (mg/g) at equilibrium and at time $t(\mathrm{~min})$, respectively. $k_{1}$ is the rate constant of adsorption $\left(\mathrm{min}^{-1}\right)$ and calculated from the plots of $\ln \left(q_{\mathrm{e}}-q_{\mathrm{t}}\right)$ versus $t$ for different concentrations of pyridine. The results of $k_{1}$ and correlation coefficients $\left(R^{2}\right)$ are shown in Table 1.

Pseudo-second-order kinetic model is based on the assumption that the rate-determining step may be a chemical sorption involving valence forces through sharing or exchange of electrons between adsorbent and adsorbate. The pseudo-second-order kinetic model is expressed as follows:

$$
\frac{t}{q_{\mathrm{t}}}=\frac{1}{k_{2} q_{\mathrm{e}}^{2}}+\frac{t}{q_{\mathrm{e}}}
$$

where $k_{2}$ is the pseudo-second-order rate constant $\left(\mathrm{g} \cdot \mathrm{mg}^{-1} \cdot \mathrm{min}^{-1}\right)$. Values of $k_{2}$ and $q_{\mathrm{e}}$ are calculated from the intercept and slope of the plots of $t / q$ versus $t$. The results of $k_{2}$ and correlation coefficients $\left(R^{2}\right)$ are shown in Table 1 .

The Elovich equation is often used to interpret the kinetics of sorption and successfully describe the predominantly chemical sorption on highly heterogeneous sorbents. It is based on kinetic principle assuming that the sorption sites increase exponentially with sorption, which implies a multilayer sorption and each layer exhibits a different activation energy for chemisorption. The Elovich equation can be written, in its integrated form, as follows: 


$$
q_{\mathrm{t}}=\frac{1}{\beta} \ln (\alpha \beta)+\frac{1}{\beta} \ln t
$$

where, $\alpha$ is the initial adsorption rate $\left(\mathrm{mg} \cdot \mathrm{g}^{-1} \cdot \mathrm{min}^{-1}\right)$ and $\beta$ is the desorption constant $\left(\mathrm{g} \cdot \mathrm{mg}^{-1}\right)$. The term $1 / \beta$ indicates the number of sites available for adsorption. A plot between $q_{\mathrm{t}}$ versus $\ln (t)$ yields a linear relationship with a slope of $(1 / \beta)$ and an intercept of $(1 / \beta) \ln (\alpha \beta)$. The values of $\alpha, \beta$ and correlation coefficients are shown in Table 1.

The intra-particle diffusion model is used to identify the diffusion mechanism. This kinetic model considers the rate limiting step of transport of adsorbates from bulk into adsorbents and is expressed as follows:

$$
q_{\mathrm{t}}=I+K_{\text {id }} \sqrt{t}
$$

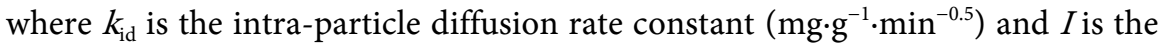
intercept of vertical axis $\left(\mathrm{mg} \cdot \mathrm{g}^{-1}\right)$. According to Equation (4), a plot of $q_{\mathrm{t}}$ versus $t_{1 / 2}$ should be a straight line with a slope $\mathrm{k}_{\mathrm{id}}$ and intercept $I$ when adsorption mechanism follows the intra-particle diffusion process. The values of $k_{\mathrm{id}}$ and correlation coefficients are listed in Table 1.

The two-constant model is a useful empirical equation. With two fitting parameters this model is quite flexible to predict the extraction kinetics, but note that this equation has not been rigorously derived or explained. The two-constant kinetic model is expressed as follows:

$$
\ln q_{\mathrm{t}}=\ln a+b \ln t
$$

where $a$ is the initial desorption rate constant $\left(\mathrm{mg} \cdot \mathrm{g}^{-1} \cdot \mathrm{min}^{-1}\right)$ and $b$ is the desorption rate coefficient $\left[\left(\mathrm{mg} \cdot \mathrm{g}^{-1}\right)^{-1}\right]$. The values of $a$ and $b$ and correlation coefficients are listed in Table 1. The fitting curves and calculated parameters of the four adsorption kinetics models at different temperatures are shown in Figure 4 and Table 1 , respectively.

Since each fitting equation has different assumptions, the correlation coefficient $\left(R^{2}\right)$ represents the correlation between the equation and the adsorption curves. It is generally believed that when the correlation coefficient $\mathrm{R}^{2}$ of the fitting equation reaches $\geq 0.99$, the adsorption curves conform to the fitting equation and the adsorption process satisfies the assumption of the fitting equation. Fitting the adsorption curve can also contribute to the prediction of adsorption equilibrium and adsorption capacity. The correlation coefficients of the pseudo-first-order model, two-constant model, intra-particle diffusion model and Elovich kinetic model are found to be less than 0.99. Furthermore, the adsorption capacities calculated using these fitting models greatly deviate from the experiment data. By contrast, the correlation coefficients of the pseudo-second-order kinetic model are more than 0.99 , suggesting that the adsorption of pyridine on shale matches well with the pseudo-second-order kinetic model. Our kinetic results for the adsorption of pyridine by shale are consistent with some previous studies using bamboo charcoal [15], mesoporous silica [16] and polymeric adsorbents [3] [17] [18]. 


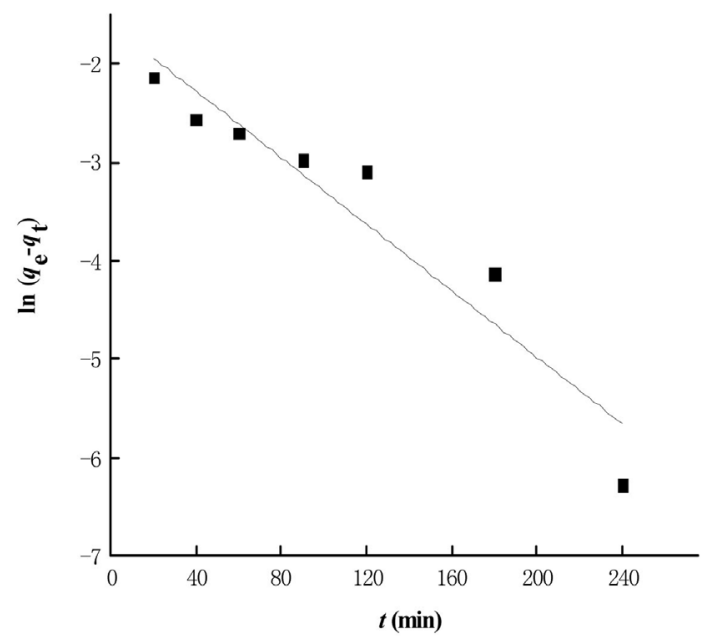

(A)

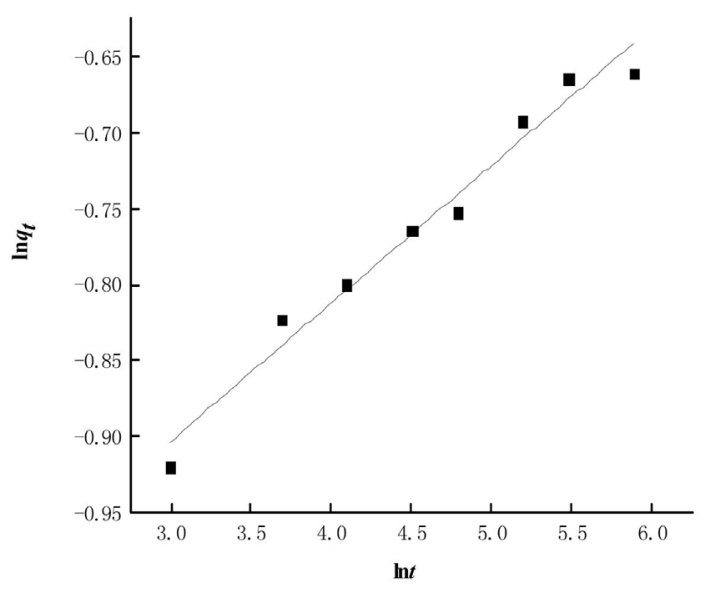

(C)

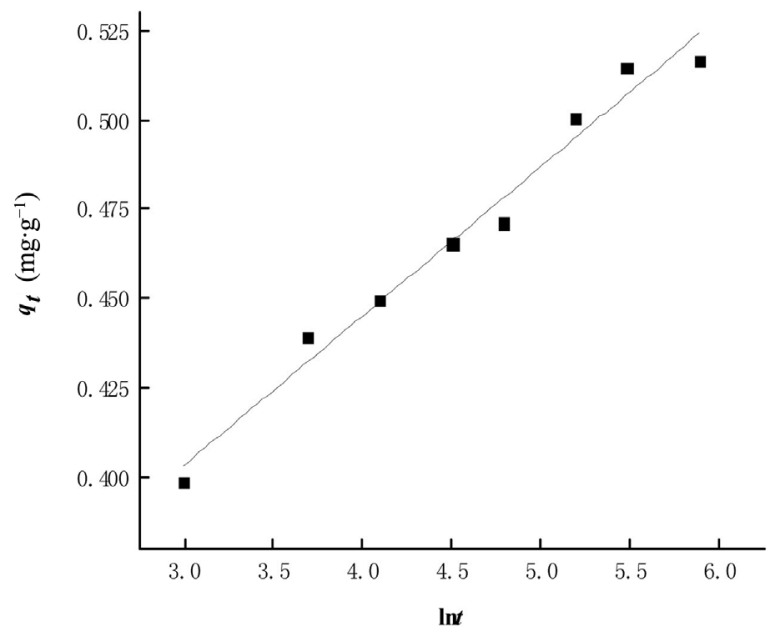

(E)

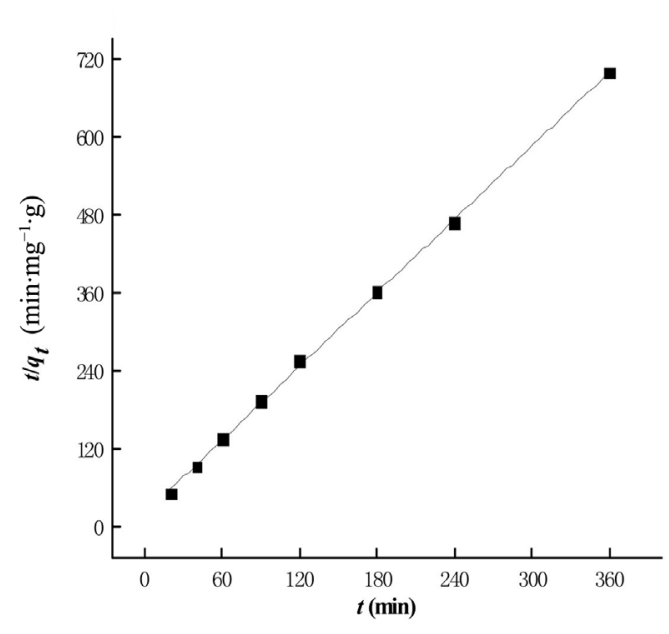

(B)

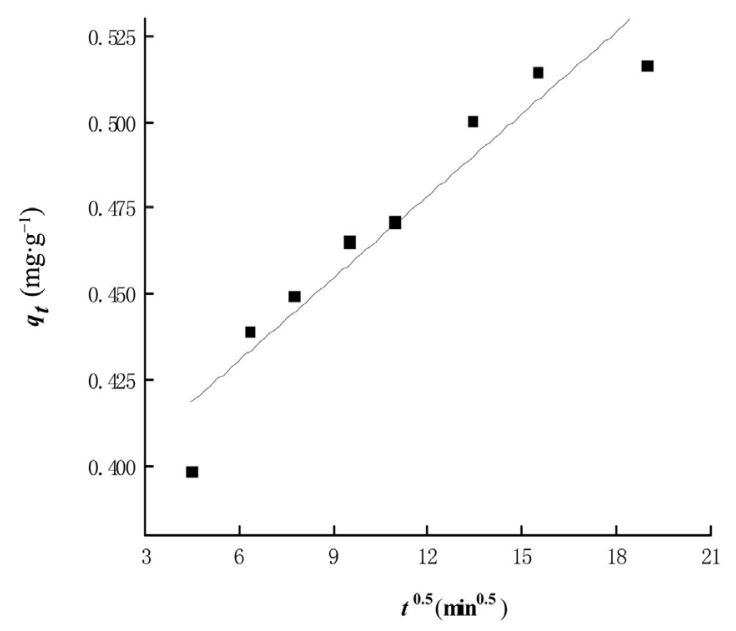

(D)

Figure 4. Various adsorption kinetics model fitting curves for the removal of pyridine by shale: (A) Pseudo-first-order model; (B) Pseudo-second-order model; (C) Two-constant model; (D) Intra-particle diffusion model; (E) Elovich model. 


\subsection{Adsorption Isotherms}

Some isotherm models are presented to describe the adsorption process such as Langmuir, Freundlich, Tempkin, and Redlich-Peterson. A survey of adsorption isotherms by Awad et al. (2019) reveals that Langmuir, Freundlich and Temkin are more frequently employed by previous researchers to investigate the simulation experiments. Based on the statistical result of Awad et al. (2019), we tentatively examine the present experimental data using Equations (6), (7) and (8) as followed.

Langmuir equation:

$$
\frac{C_{\mathrm{e}}}{q_{\mathrm{e}}}=\frac{C_{\mathrm{e}}}{q_{\max }}+\frac{1}{K_{\mathrm{L}} \times q_{\max }}
$$

Freundlich equation:

$$
\lg q_{\mathrm{e}}=\frac{\lg C_{\mathrm{e}}}{n}+\lg K_{\mathrm{F}}
$$

Tempkin isotherm is represented by the following equation:

$$
q_{\mathrm{e}}=\frac{R T}{b_{\mathrm{T}}} \ln K_{\mathrm{T}}+\frac{R T}{b_{\mathrm{T}}} \ln C_{\mathrm{e}}
$$

where $C_{\mathrm{e}}$ stands for equilibrium concentration of pyridines $(\mathrm{mg} / \mathrm{L}), q_{\mathrm{e}}$ denotes the equilibrium adsorption capacity (mg/g), $q_{\max }$ refers to maximum adsorption capacity $(\mathrm{mg} / \mathrm{g}), K_{\mathrm{L}}$ designates Langmuir constant $(\mathrm{L} / \mathrm{mg}), K_{\mathrm{F}}$ represents the Freundlich constant $(\mathrm{mg} / \mathrm{g})(\mathrm{mg} / \mathrm{L})^{(-1 / n)}, K_{\mathrm{T}}$ signifies the equilibrium binding constant corresponding to the maximum binding energy, and $b_{\mathrm{T}}$ is equilibrium Temkin binding constant, related to the heat of adsorption. The fitting curves of the Langmuir, Freundlich and Temkin isotherms were exhibited in Figure 5.

The related model parameters and the corresponding regression coefficients are listed in Table 2. The Freundlich model with the highest $R^{2}$ values (Table 2) demonstrates its best describing the whole process for pyridine removal in comparison with the Langmuir and Temkin models.

Table 2. Parameters of adsorption isotherm models.

\begin{tabular}{cccc}
\hline Model & Parameters & $298 \mathrm{~K}$ & $\mathrm{R}^{2}$ \\
\hline \multirow{2}{*}{ Langmuir } & $K_{L}(\mathrm{~L} / \mathrm{mg})$ & 0.003 & 0.984 \\
& $q_{\max }(\mathrm{mg} / \mathrm{g})$ & 3.508 & \\
\multirow{2}{*}{ Freundlich } & $K_{F}(\mathrm{mg} / \mathrm{g})(\mathrm{mg} / \mathrm{L})^{(-1 / \mathrm{n})}$ & 0.008 & 0.994 \\
& $\mathrm{n}$ & 1.103 & \\
\multirow{2}{*}{ Temkin } & $K_{T}$ & 0.047 & 0.856 \\
\hline
\end{tabular}

\subsection{Comparison with Other Studies Using Natural and/or Synthetic Materials}

It should be noted that some previous researchers have carried out laboratory experiments to examine the performance of natural and/or synthetic materials as adsorbents for pyridine removal (Table 3). From Table 3 it could be concluded that the Langmuir and Freundlich models are regularly utilized to test the isothermal experimental data. 


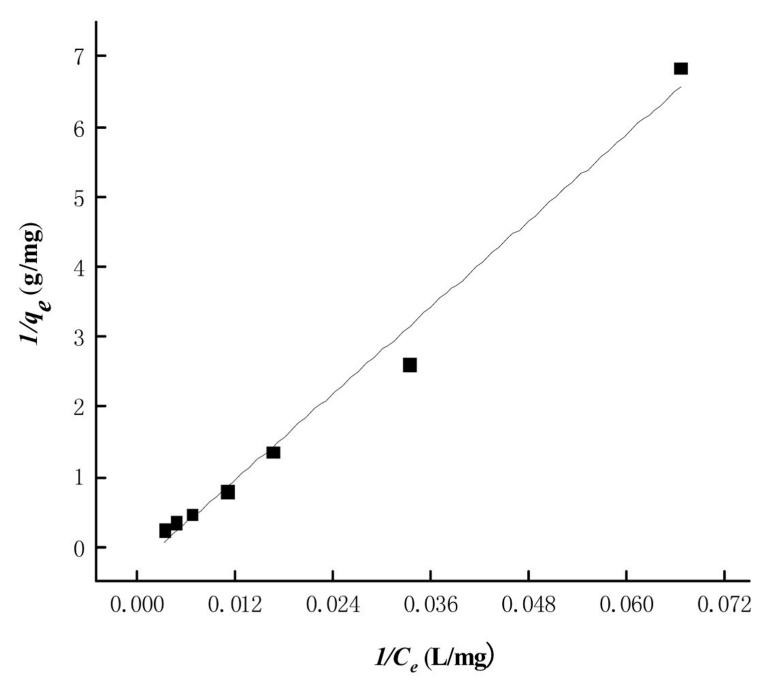

(A)

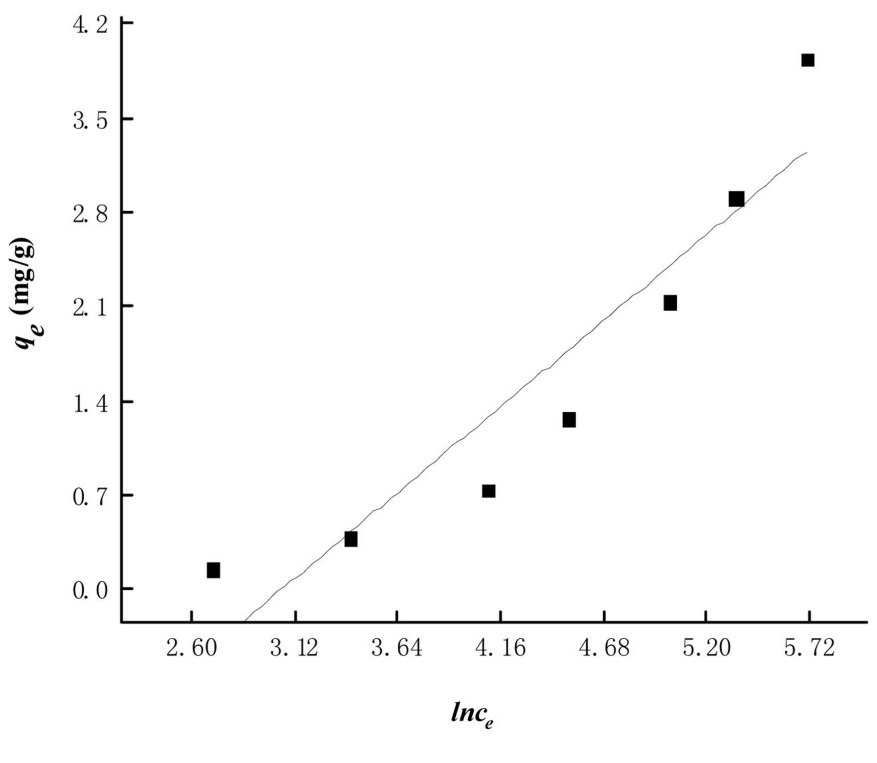

(C)

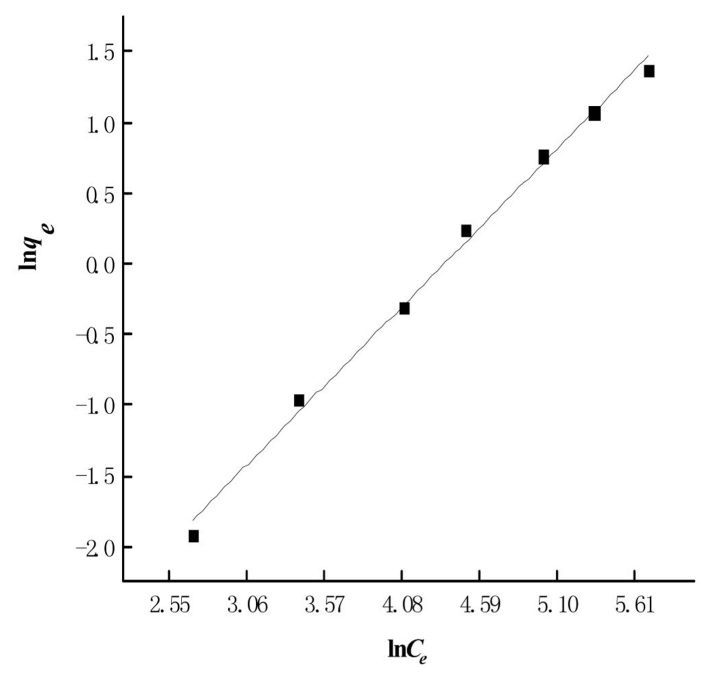

(B)

Figure 5. Adsorption isotherms of pyridine on shale: (A) Langmuir model; (B) Freundlich model; (C) Temkin model.

Our investigation reveals that the removal of pyridine by shale from aqueous solution is much better demonstrated by the Freundlich adsorption isotherm model than the Langmuir model. This result coincides with former studies employing minerals widespread in sedimentary basins including natural apatite [24], kaolinite [25], and montmorillonite [25]. Our preliminary study shows that natural shale exhibits a limited adsorption capacity (of an order of magnitude of $1 \mathrm{mg} / \mathrm{g}$ ) in Table 2 . Nevertheless, unmodified shale may be potentially applied to treat wastewaters containing trace pyridines. Should the adsorption capacity of the natural shale be further improved by different modifications in the next wok, natural shale is undoubtedly one of the promising candidates for pyridine bearing effluents treatment since it is environment-friendly, cost-effectively and rich in nature. 
Table 3. Isothermal models for removal of pyridine by natural and/or synthetic adsorbents.

\begin{tabular}{|c|c|c|}
\hline Adsorbents & Isotherm models & References \\
\hline$\alpha-\mathrm{Al}_{2} \mathrm{O}_{3}$ & Langmuir, FFG & [19] \\
\hline Apatite & Freundlich & [20] \\
\hline Activated carbon & Langmuir & [7] \\
\hline Activated carbon & Toth and Radke-Prausnitz & [4] \\
\hline Activated carbon fibers & Langmuir & [8] \\
\hline Activated carbon cloth & Prausnitz-Radke & [21] \\
\hline Bagasse fly ash & Langmuir & {$[2]$} \\
\hline Bamboo charcoal & Freundlich & [16] \\
\hline Carbon nanotube & Freundlich & [22] \\
\hline Ion-exchange resin & Langmuir, Freundlich & [23] \\
\hline Iron powder & Langmuir, FFG & [19] \\
\hline Kaolinite & Freundlich & {$[24]$} \\
\hline Mesoporous silica & Langmuir & {$[17]$} \\
\hline Montmorillonite & Freundlich & {$[24]$} \\
\hline Poly 4-vinyl aniline-co-DVB & Freundlich & [25] \\
\hline Polymeric adsorbents & Langmuir, Freundlich & {$[3]$} \\
\hline Post-crosslinked fiber & Freundlich & {$[18]$} \\
\hline Rice husk ash & Redlich-Peterson & {$[4]$} \\
\hline Spent oil shale & Langmuir & [26] \\
\hline Zeolite & Langmuir-Freundlich & [27] \\
\hline Shale & Freundlich & This study \\
\hline
\end{tabular}

\section{Conclusion}

Preliminary laboratory experiments are carried out to investigate the performance of natural shale from Yichang, China as an adsorbent for pyridine removal from wastewaters. It is found that the prepared shale samples with well-developed porosity are mainly composed of illite, quartz, calcite, chlorite and sericite. The kinetics of the adsorption processes conforms to a pseudo-second-order rate law with the adsorption data characterized by the Freundlich isotherm model. The preliminary results denote that natural shale collected from sedimentary basins may be employed as one of the potential low-cost adsorbents for treating effluents containing trace pyridine. Modification methods of shale still need further investigation in the next work.

\section{Acknowledgements}

We gratefully acknowledge financial support by the National Natural Science Foundation of China (No. 41730424; 41472095) and the Open Fund of Key Laboratory of Exploration Technologies for Oil and Gas Resources (Yangtze Uni- 
versity), Ministry of Education (No. K2018-05).

\section{Conflicts of Interest}

The authors declare no conflicts of interest regarding the publication of this paper.

\section{References}

[1] Padoley, K.V., Mudliar, S.N. and Pandey, R.A. (2008) Heterocyclic Nitrogenous Pollutants in the Environment and Their Treatment Options-An Overview. Bioresource Technology, 99, 4029-4043. https://doi.org/10.1016/j.biortech.2007.01.047

[2] Lataye, D.H., Mishra, I.M. and Mall, I.D. (2006) Removal of Pyridine from Aqueous Solution by Adsorption on Bagasse Fly Ash. Industrial and Engineering Chemistry Research, 45, 3934-3943. https://doi.org/10.1021/ie051315w

[3] Zhu, Q., Moggridge, G.D. and Ainte, M. (2016) Adsorption of Pyridine from Aqueous Solutions by Polymeric Adsorbents MN 200 and MN 500. Part 1: Adsorption Performance and PFG-NMR Studies. Chemical Engineering Journal, 306, 67-76. https://doi.org/10.1016/j.cej.2016.07.039

[4] Lataye, D.H., Mishra, I.M. and Mall, I.D. (2008) Pyridine Sorption from Aqueous Solution by Rice Husk Ash (RHA) and Granular Activated Carbon (GAC): Parametric, Kinetic, Equilibrium and Thermodynamic Aspects. Journal of Hazardous Materials, 154, 858-870. https://doi.org/10.1016/j.jhazmat.2007.10.111

[5] Subbaramaiah, V., Srivastava, V.C. and Mall, I.D. (2013) Catalytic Wet Peroxidation of Pyridine Bearing Wastewater by Cerium Supported SBA-15. Journal of Hazardous Materials, 248, 355-363. https://doi.org/10.1016/j.jhazmat.2013.01.018

[6] Singh, N.B., Nagpal, G. and Agrawal, S. (2018) Water Purification by Using Adsorbents: A Review. Environmental Technology and Innovation, 11, 187-240. https://doi.org/10.1016/j.eti.2018.05.006

[7] Mohan, D., Singh, K.P., Sinha, S. and Gosh, D. (2004) Removal of Pyridine from Aqueous Solution Using Low Cost Activated Carbons Derived from Agricultural Waste Materials. Carbon, 42, 2409-2421.

https://doi.org/10.1016/j.carbon.2004.04.026

[8] Li, B., Lei, Z., Zhang, X. and Huang, Z. (2010) Adsorption of Simple Aromatics from Aqueous Solutions on Modified Activated Carbon Fibers. Catalysis Today, 158, 515-520. https://doi.org/10.1016/j.cattod.2010.08.014

[9] Ahmaruzzaman, M. (2008) Adsorption of Phenolic Compounds on Low-Cost Adsorbents: A Review. Advances in Colloid and Interface Science, 143, 48-67. https://doi.org/10.1016/j.cis.2008.07.002

[10] Lin, S.H. and Juang, R.S. (2009) Adsorption of Phenol and Its Derivatives from Water Using Synthetic Resins and Low-Cost Natural Adsorbents: A Review. Journal of Environmental Management, 90, 1336-1349. https://doi.org/10.1016/j.jenvman.2008.09.003

[11] Tang, X., Ripepi, N., Rigby, S., Mokaya, R. and Gilliland, E. (2019) New Perspectives on Supercritical Methane Adsorption in Shales and Associated Thermodynamics. Journal of Industrial and Engineering Chemistry, 78, 186-197. https://doi.org/10.1016/j.jiec.2019.06.015

[12] Rouxhet, P.G., Samudacheata, N., Jacobs, H. and Anton, O. (1977) Attribution of the OH Stretching Bands of Kaolinite. Clay Minerals, 12, 171-179.

https://doi.org/10.1180/claymin.1977.012.02.07 
[13] Ding, K., Li, S., Yue, C. and Zhong, N. (2007) Simulation Experiments on Thermochemical Sulfate Reduction Using Natural Gas. Journal of Fuel Chemistry and Technology, 35, 401-406. https://doi.org/10.1016/S1872-5813(07)60025-5

[14] Więckowski, T. and Wiewióra, A. (1976) New Approach to the Problem of the Interlayer Bonding in Kaolinite. Clays and Clay Minerals, 24, 219-223. https://doi.org/10.1346/CCMN.1976.0240502

[15] Awad, A.M., Shaikh, S.M.R., Jalab, R., Gulied, M.H., Nasser, M.S., Benamor, A. and Adham, S. (2019) Adsorption of Organic Pollutants by Natural and Modified Clays: A Comprehensive Review. Separation and Purification Technology, 228, Article ID: 115719. https://doi.org/10.1016/j.seppur.2019.115719

[16] Liao, P., Yuan, S., Xie, W., Zhang, W., Tong, M. and Wang, K. (2013) Adsorption of Nitrogen-Heterocyclic Compounds on Bamboo Charcoal: Kinetics, Thermodynamics, and Microwave Regeneration. Journal of Colloid and Interface Science, 390, 189-195. https://doi.org/10.1016/j.jcis.2012.09.037

[17] Singh, K., Chandra, B., Rhyman, L. and Ramasami, P. (2016) Effective Adsorption of Pyridine (Py)—Onto Mesoporous Silica Derived from De-Oiled Mustard Cake (DOMC): Experimental and Theoretical Study. Journal of Environmental Chemical Engineering, 4, 1383-1392. https://doi.org/10.1016/j.jece.2016.01.033

[18] Zhang, Y. and Li, D. (2010) Adsorption of Pyridine on Post-Crosslinked Fiber. Journal of Scientific and Industrial Research, 69, 73-76.

[19] Ardizzone, S., Høiland, H., Lagioni, C. and Sivieri, E. (1998) Pyridine and Aniline Adsorption from an Apolar Solvent: The Role of the Solid Adsorbent. Journal of Electroanalytical Chemistry, 447, 17-23. https://doi.org/10.1016/S0022-0728(98)00007-2

[20] Bouyarmanea, H., Ei Asria, S., Ramib, A., Rouxc, C., Mahlyb, M.A., Saoiabia, A., Coradinc, T. and Laghzizila, A. (2010) Pyridine and Phenol Removal Using Natural and Synthetic Apatites as Low Cost Sorbents. Influence of Porosity and Surface Interactions. Journal of Hazardous Materials, 181, 736-741.

https://doi.org/10.1016/j.jhazmat.2010.05.074

[21] Alonso-Davila, P., Torres-Rivera, O.L., Leyva-Ramos, R. and Ocampo-Perez, R. (2012) Removal of Pyridine from Aqueous Solution by Adsorption on an Activated Carbon Cloth. Clean-Soil Air Water, 40, 45-53.

https://doi.org/10.1002/clen.201100049

[22] Zhao, B., Liang, H., Han, D., Qiu, D. and Chen, S. (2007) Adsorption of Pyridine from Aqueous Solution by Surface Treated Carbon Nanotubes. Separation Science and Technology, 42, 3419-3427. https://doi.org/10.1080/01496390701511689

[23] Akita, S. and Takeuchi, H. (1993) Sorption Equilibria of Pyridine Derivatives in Aqueous Solution on Porous Resins and Ion Exchange Resins. Journal of Chemical Engineering of Japan, 26, 237-241. https://doi.org/10.1252/jcej.26.237

[24] Baker, R.A. and Luh, M.D. (1971) Pyridine Sorption from Aqueous Solution by Montmorillonite and Kaolinite. Water Research, 5, 839-848. https://doi.org/10.1016/0043-1354(71)90020-0

[25] Mathidala, S. and Ogunlaja, A.S. (2019) Selective Removal of Pyridine in Fuel by Imprinted Polymer (Poly 4-vinyl aniline-co-DVB) as Adsorbent. Petroleum Science and Technology, 37, 1691-1703. https://doi.org/10.1080/10916466.2019.1602641

[26] Zhu, S., Bell, P.R.F. and Greenfield, P.F. (1988) Isotherm Studies on Sorption of Pyridine and Quinoline onto Rundle Spent Shale. Fuel, 67, 1316-1320. https://doi.org/10.1016/0016-2361(88)90110-X 
[27] Bludau, H., Karge, H.G. and Niessen, W. (1998) Sorption, Sorption Kinetics and Diffusion of Pyridine in Zeolites. Microporous and Mesoporous Materials, 22, 297-308. https://doi.org/10.1016/S1387-1811(98)00093-6 